\title{
A Case of Intestinal Tuberculosis Mimicking Crohn's Disease: A Clinical and Diagnostic Dilemma
}

\author{
Sharon Esther Weinberg, Ahsan M Mughal \\ Department of Respiratory Medicine, Morriston Hospital, Swansea Bay University Health Board, Swansea, Wales, UK
}

Doi: 10.12890/2021_002699 - European Journal of Case Reports in Internal Medicine - ๑ EFIM 2021

Received: 07/06/2021

Accepted: 05/10/2021

Published: 09/11/2021

How to cite this article: Weinberg SE, Mughal AM. A case of intestinal tubercolosis mimicking Chron's disease: a clinica and diagnostic dilemma. EJCRIM 2021;8: doi:10.12890/2021_002699.

Conflicts of Interests: The authors declare there are no competing interests.

This article is licensed under a Commons Attribution Non-Commercial 4.0 License

\section{ABSTRACT}

This case highlights the importance of differentiating between Crohn's disease and intestinal tuberculosis. The rates of misdiagnosis of Crohn's disease and intestinal tuberculosis range from $50 \%$ to $70 \%$ because of their non-specific and clinically similar manifestations.If intestinal tuberculosis is misdiagnosed as Crohn's disease, use of immunomodulatory drugs commonly used for Crohn's disease can increase the risk of disseminated tuberculosis. Here we present a case highlighting the clinical similarity between these two distinct medical conditions and suggest how a similar scenario can be approached, which can help to differentiate between the two otherwise very similar conditions.

\section{LEARNING POINTS}

- $\quad$ Given the similarities, it is key to differentiate Crohn's disease from intestinal tuberculosis as early as possible.

- Patients undergoing colonoscopy for possible Crohn's disease should have colonic biopsy samples sent for AFB culture.

- Consider investigations for intestinal tuberculosis in uncontrolled Crohn's disease where intestinal tuberculosis has not been worked up previously.

\section{KEYWORDS}

Tuberculosis, inflammatory bowel disease

\section{CASE DESCRIPTION}

A 46-year-old British-born NHS doctor of Indian descent was referred to the chest clinic for consideration of treatment for latent tuberculosis prior to the initiation of anti-tumour necrosis factor-alpha (anti TNF- $\alpha$ ) therapy for Crohn's disease. He had been diagnosed with Crohn's disease 4 years earlier following the development of a perianal abscess for which he underwent surgical drainage. He had been reviewed by the gastroenterologist and was prescribed mesalazine and budesonide which he took intermittently with partial symptomatic improvement. He was considered for anti-TNF- $\alpha$ therapy and as a QuantiFERON ${ }^{\circledR}$ test returned positive, he was referred to the chest clinic. In the chest clinic, the patient denied any respiratory symptoms and his chest x-ray was normal. During this consultation, symptoms, investigations and radiology were reviewed. He reported abdominal pain, cramps, loose stools and weight loss. He was born and raised in Swansea, Wales, and had no exposure to unpasteurized milk. He had no recent travel in the past 5 years. His previous perianal abscess cultures showed mixed anaerobes, so he was treated with a prolonged course of oral antibiotics. Colonoscopy revealed patchy inflammation in the transverse and ascending colon. Histological samples showed focal granuloma formations which were acid-fast (Ziehl-Neelsen) negative. A magnetic resonance enterogram reported small bowel and caecal thickening, which was thought to be consistent with Crohn's disease. 
Given the patient's history and relatively poor response to treatment, the decision to treat for latent tuberculosis was withheld and the patient was referred for an urgent colonoscopy for an intestinal biopsy for Mycobacterium cultures, which confirmed intestinal tuberculosis, sensitive to first-line anti-tuberculosis drugs.

After discussion with the gastroenterology team, the patient's mesalazine and budesonide were stopped and he was started on standard quadruple anti-tuberculosis treatment with complete resolution of his symptoms. A repeat magnetic resonance enterogram after intestinal tuberculosis treatment showed resolution of previous radiological changes. Due to clinical and radiological improvement, a joint decision was made not to perform any further endoscopy.

\section{DISCUSSION}

Crohn's disease is a chronic relapsing and remitting gastrointestinal inflammatory disorder with unclear aetiology ${ }^{[1]}$. In contrast, intestinal tuberculosis can be cured with a 6-month course of chemotherapy ${ }^{[2]}$. Both diseases have many clinical, endoscopic, histological and radiological similarities ${ }^{[1,2]}$. Correct diagnosis is important as the use of steroids, immunosuppressive agents and TNF- $\alpha$ therapy in Crohn's disease may have disastrous effects on patients with tuberculosis ${ }^{[1,3]}$.

A variety of clinical, endoscopic, histological and radiological criteria have been recommended to assist with differentiating intestinal tuberculosis from Crohn's disease (Table 1) ${ }^{[2,4,5]}$. Endoscopic differentiation includes anorectal lesions, a cobblestone appearance, longitudinal ulcers and aphthous ulcers, all more common in Crohn's disease, while transverse ulcers and pseudopolyps are more common in intestinal tuberculosis ${ }^{[2,5]}$. The endoscopic macroscopic appearance will show some overlap in $60-70 \%$ of patients ${ }^{[2]}$.

Radiographically, a bowel wall with symmetrical and circumferential thickening along with strictures and fistulas is more common in Crohn's disease, while necrotic lymph nodes are more common in intestinal tuberculosis ${ }^{[2,5]}$. In clinical practice, however, most of these parameters lack specificity or only exist during specific stages of the diseases ${ }^{[4]}$.

\begin{tabular}{|c|c|c|}
\hline & Crohn's disease & Intestinal tuberculosis \\
\hline Clinical manifestation & $\begin{array}{l}\text { Chronic relapsing and remitting; aphthous ulcers; } \\
\text { fatigue, bloody stools; perianal disease }{ }^{[1]} \text {, weight } \\
\text { loss, arthropathies }{ }^{[1]}\end{array}$ & $\begin{array}{l}\text { Symptoms of months to years, fever, night sweats; } \\
\text { abdominal pain ( } 81 \%) \text {, weight loss (46\%), vomiting } \\
(22 \%) \text {, loose stools }(14 \%)^{[1,6,7]}\end{array}$ \\
\hline $\begin{array}{l}\text { Common anatomical } \\
\text { site }\end{array}$ & $\begin{array}{l}\text { Entire gastrointestinal tract, rectum, terminal ileum } \\
{[1,4]}\end{array}$ & Ileum, ileocecal valve, right colon ${ }^{[4,7]}$. \\
\hline $\begin{array}{l}\text { Radiographic findings } \\
\text { (CT/MRI) }\end{array}$ & $\begin{array}{l}\text { Symmetrical and circumferential thickening with } \\
\text { strictures and fistulas }{ }^{[2]}\end{array}$ & $\begin{array}{l}\text { Necrotic lymph nodes, asymmetrical mesenteric } \\
\text { thickening, bowel wall thickening, ascites }{ }^{[2]}\end{array}$ \\
\hline Endoscopic findings & $\begin{array}{l}\text { Anorectal lesions, skip lesions, cobblestone } \\
\text { appearance, longitudinal and aphthous ulcers }{ }^{[1,8]}\end{array}$ & $\begin{array}{l}\text { Circumferential ulcers, pseudopolyps, hypertrophic } \\
\text { mucosa, strictures } \\
{[1,2]}\end{array}$ \\
\hline $\begin{array}{l}\text { Histopathological } \\
\text { findings }\end{array}$ & $\begin{array}{l}\text { Poorly organized small granulomas, more commonly } \\
\text { in rectosigmoid disease }{ }^{[1]}\end{array}$ & $\begin{array}{l}\text { Granulomas with caseating necrosis, conglomerate } \\
\text { epithelioid histiocytes and disproportionate } \\
\text { submucosal inflammation (13-33\%) }\end{array}$ \\
\hline Management & Lifelong immunosuppression & 6-Month chemotherapy \\
\hline
\end{tabular}

Table 1. Diagnostic parameters

In intestinal tuberculosis, caseous granulomas and culture positive for Mycobacterium tuberculosis have conventionally been regarded as the gold standard for diagnosis, although often limited by poor sensitivity ${ }^{[1]}$. Granulomas with or without caseation are seen in less than $50 \%$ of resection specimens and even less frequently in biopsy samples of intestinal tuberculosis (15-36\%) ${ }^{[1,4]}$. 
The classic histological feature of granulomas with caseating necrosis, conglomerate epithelioid histiocytes and disproportionate submucosal inflammation are seen in only $13-33 \%$ of patients ${ }^{[5]}$ and positive culture is seen in only $20-53 \%$ of cases ${ }^{[6]}$. Tuberculosis polymerase chain reaction (PCR) analysis has been shown to be more sensitive than culture and acid-fast staining in diagnosing intestinal tuberculosis, with a sensitivity of up to $66 \%{ }^{[5]}$ and a specificity of up to $90 \%{ }^{[4]}$.

We suggest that in all patients with a clinical question of possible Crohn's disease, the clinician perform the following steps, along with appropriate clinical assessment, to help differentiate Crohn's disease from intestinal tuberculosis (Fig. 1): (1) send up to eight intestinal biopsy samples for acid-fast bacilli (AFB) culture ${ }^{[6]}$ and if possible, tuberculosis PCR testing; (2) look for necrotic lymphadenopathy on cross-sectional imaging and histological features suggestive of intestinal tuberculosis, particularly caseation; (3) perform high generation interferon gamma release assay (IGRA) testing (QuantiFERON-TB Gold Plus or T-SPOT.TB test). A positive step 1 along with supportive clinical and/or radiological features suggestive of tuberculosis confirms intestinal tuberculosis even if the remaining two steps are not fully supportive of the diagnosis of intestinal tuberculosis. If step 1 is negative but any features of step 2 are positive, this is supportive of a diagnosis of intestinal tuberculosis. In countries with a high incidence of tuberculosis, therapeutic anti-tubercular therapy (ATT) trials might be considered at this point in patients without drug sensitivities, however we suggest repeating biopsy for cultures (step 1) to rule out drug resistance. If only step 3 is positive, the patient is more likely to have Crohn's disease and should be considered for latent tuberculosis treatment after appropriate work-up and excluding active tuberculosis at other sites. If all three steps are negative for tuberculosis, Crohn's disease is the likely diagnosis.

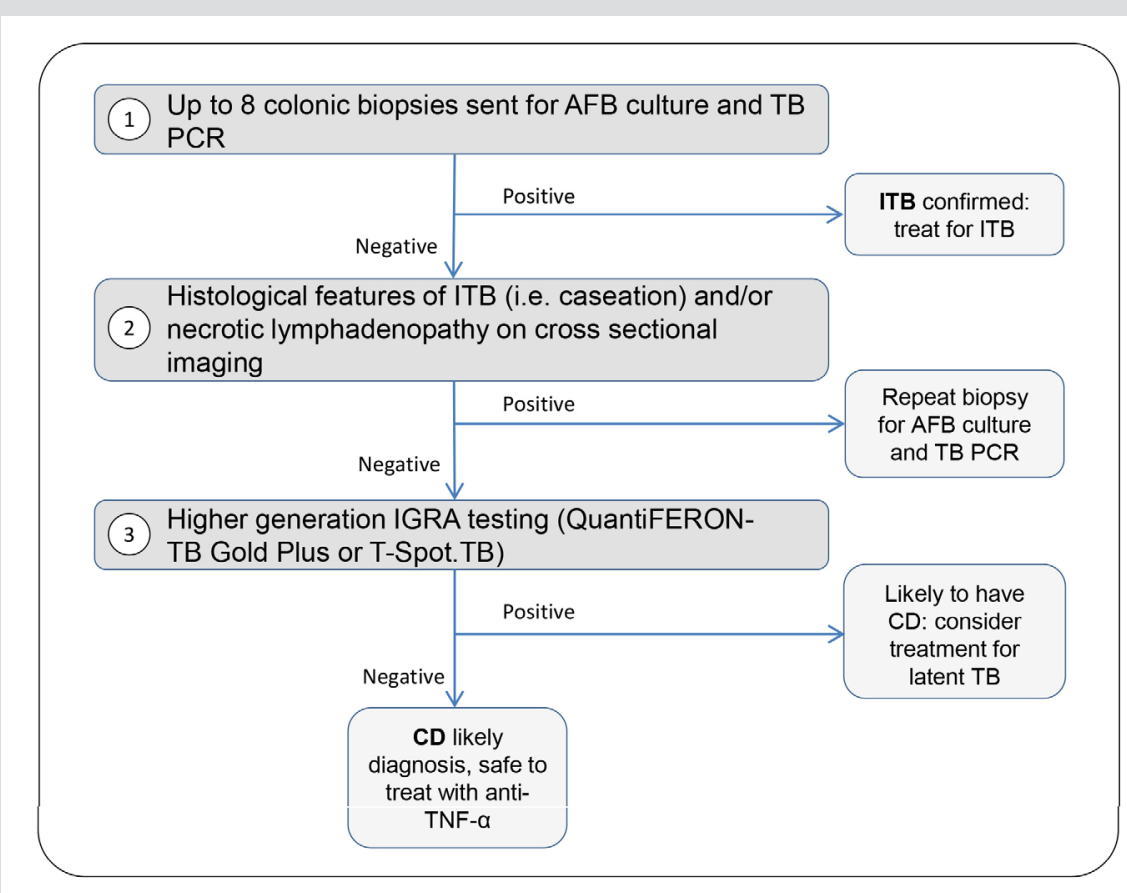

Figure 1. Proposed investigation strategy to differentiate intestinal tuberculosis (ITB) from Crohn's disease (CD). *After appropriate work-up and excluding active TB at other sites. ITB, intestinal tuberculosis

In countries with a low incidence of tuberculosis like the United Kingdom, histological samples from patients undergoing colonoscopy for suspected Crohn's disease are usually fixed in formalin and are not routinely sent for AFB culture. However, if latent or active tuberculosis is suspected when anti-TNF- $\alpha$ treatment is being considered, the patient may require another colonoscopy so histological samples can be sent for AFB culture in a non-formalin solution, as in our case. We suggest routinely sending samples in saline for AFB culture, and tuberculosis PCR if possible, for all suspected Crohn's disease patients so the correct diagnosis can be made and a repeat procedure avoided. We believe the cost and risk of prolonging treatment to take extra specimens are minimal and offset by having the correct diagnosis and reducing the need for a repeat procedure in the future. We also suggest that patients with 'uncontrolled' Crohn's disease despite treatment, where intestinal biopsies for AFB culture have not been sent previously, should be considered for repeat colonoscopy for intestinal tuberculosis work-up. We believe that such an approach may reveal some patients misdiagnosed with intestinal tuberculosis. 


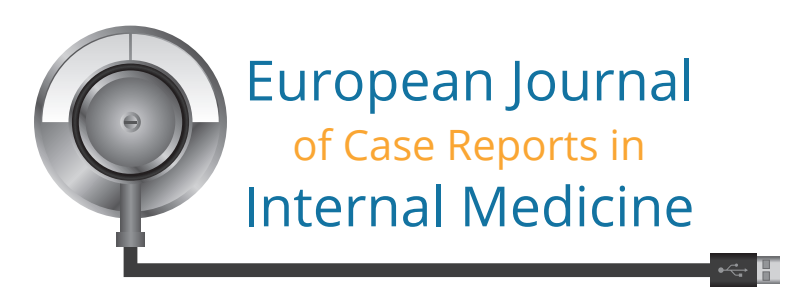

\section{REFERENCES}

1. Ma JY, Tong JL, Ran ZH. Intestinal tuberculosis and Crohn's disease: challenging differential diagnosis. J Dig Dis 2016;17(3):155-161.

2. Epstein D, Watermeyer G, Kirsch R. Review article: the diagnosis and management of Crohn's disease in populations with high-risk rates for tuberculosis. Aliment Pharmacol Ther 2007;25(12):1373-1388.

3. Dobler CC. Biologic agents and tuberculosis. In: Schlossberg D, editor. Tuberculosis and nontuberculous mycobacterial infections. 7th ed. Washington, DC: ASM Press; 2017, pp. 623-635.

4. Zhao XS, Wang ZT, Wu ZY, Yin QH, Zhong J, Miao F, et al. Differentiation of Crohn's disease from intestinal tuberculosis by clinical and CT enterographic models. Inflamm Bowel Dis 2014;20(5):916-925.

5. Mehta V, Desai D, Abraham P, Rodrigues C. Making a positive diagnosis of intestinal tuberculosis with the aid of new biologic and histologic features: how far have we reached? Inflamm Intest Dis 2019;3(4):155-160.

6. Mehta V, Desai D, Abraham P, Gupta T, Rodrigues C, Joshi A, et al. Do additional colonoscopic biopsies increase the yield of Mycobacterium tuberculosis culture in suspected ileo-colonic tuberculosis? Indian J Gastroenterol 2018;37(3):226-230.

7. Debi U, Ravisankar V, Prasad KK, Sinha SK, Sharma AK. Abdominal tuberculosis of the gastrointestinal tract: revisited. World J Gastroenterol 2014;20(40):14831-14840.

8. Kedia S, Das P, Madhusudhan KS, Dattagupta S, Sharma R, Sahni P, et al. Differentiating Crohn's disease from intestinal tuberculosis. World J Gastroenterol 2019;25(4):418432. 